Satwant K. Sanra MD, Uma Pandit MD, Sujit K. Pandit MD, Sarla P. Kothary MD

\title{
Effect of halogenated anaesthetics on heart rate changes during reversal of neuro- muscular block with glycopyrrolate and neostigmine
}

The effect of halothane and enflurane on changes in heart rate during reversal of neuromuscular blockade was compared in 48 parients. Premedication and anaesthetic technique was standardized. Either halothane or enfurane was used as the primary anaesthetic. Neuromuscular block was reversed by injection of glycopyrrolate and neostigmine, given either separately or simultaneously. Heart rate changes were significantiy different between the halothane and enflurane subgroups. Patients anaesthetized with enflurane showed less fluctuations in heart rate. Simultaneous injection of glycopyrrolate and neostigmine minimized the tachycardia in the halothane subgroups only.

Key words

HEART: pulse rate; ANAESTHETICS, VOLATILE: halothane, enflurane; ANTAGONISTS, NEUROMUSCULAR RELAXANTS: glycopyrrolate, neostigmine.
Changes in heart rate and rhythm during reversal of neuromuscular block have been a subject of clinical interest and investigation for many years. A number of studies have compared the effect of commonly used anticholinergics (atropine and glycopyrrolate) $^{1,2}$ and anticholinesterases (neostigmine, pyridostigmine, and edrophonium). ${ }^{3-5}$ Most of these studies have ignored any possible effect that the inhalation anaesthetics used during surgery might have had during reversal of neuromuscular block. Modification of the chronotropic effect of anticholinergic drugs by inhalation anaesthetics has been reported in man, both in paediatric ${ }^{6}$ and adult ${ }^{7}$ age groups. Recently ${ }^{8}$ Samra et al. have shown that volatile anaesthetics used during surgery have a significant modifying effect on changes in heart rate during administration of atropine and neostigmine. The present study reports results of observations when glycopyrrolate rather than atropine is used as the anticholinergic agent with neostigmine. This clinical investigation was not designed to ascertain the mechanism of action of the various drugs used.

\section{Methods}

Institutional approval of the protocol was obtained. Forty-eight healthy (ASA physical status Class I and II) patients undergoing elective surgery requiring the use of non-depolarizing muscle relaxants were studied. Surgical procedures included major gynaecological surgery in 29 , operations on bowel and biliary tract in 12, thoracotomy in four, transphenoidal hypophysectomy in two, and lumbar laminectomy in one patient. All patients were 
TABLE I Demographic data

\begin{tabular}{lcllll}
\hline & & & & \multicolumn{2}{c}{ ASA Class } \\
\cline { 5 - 6 } Sub-group & Age in years & Height in cm & Weigh in kg & $I$ & $I I$ \\
\hline HG & $33.1 \pm 14.9$ & $162.9 \pm 8.5$ & $75.2 \pm 17.4$ & 6 & 6 \\
& $(19-65)$ & $(152.5-177.5)$ & $(53.0-108)$ & & \\
EG & $32.9 \pm 13.6$ & $163.9 \pm 8.3$ & $68.0 \pm 13.5$ & 5 & 5 \\
\multirow{2}{*}{ HGN } & $(20-61)$ & $(150-177.5) \cdots$ & $(52.7-96.4)$ & & \\
\multirow{2}{*}{ EGN } & $36.34 \pm 14.9$ & $165.5 \pm 13.0$ & $67.0 \pm 17.6$ & 7 & 5 \\
& $(19-67)$ & $(152.5-185)$ & $(50.5-95.5)$ & & \\
ANOVA p-value & $42.4 \pm 17.6$ & $163.1 \pm 8.7$ & $65.6 \pm 14.6$ & 5 & 7 \\
\hline
\end{tabular}

Values are expressed as mean \pm SD. Range is shown in parentheses. Column five shows distribution of cases according to ASA physical status classification in different sub-groups. One patient in sub-group EGN was 74 years old. Ages of remaining patients in this sub-group ranged between 20 and 65 ycars.

free of cardiovascular disease. The reasons for assignment to Class II included the presence of obesity, a prolonged history of smoking, or mild hypertension controlled with diuretics only. The patients' ages ranged from 19 to 74 years. Demographic data, including distribution according to ASA classification, are given in Table I. Premedication was by diazepam, $10 \mathrm{mg}$, given orally one hour prior to induction. Patients were assigned randomly to one of the four sub-groups described below. Anaesthesia was induced with thiopentone and maintained either with halothane (Group $\mathrm{H}, \mathrm{n}$ $=24$ ) or enflurane (Group E, $n=24$ ) in a mixture of nitrous oxide 60 per cent and oxygen 40 per cent. Pancuronium was used to provide muscle relaxation and ventilation was controlled. Duration of anaesthesia varied from $1 \frac{1 / 2}{2}$ to 8 hours. Each group of patients was divided into two subgroups of 12 each according to the mode of reversal of neuromuscular block. Twelve patients in each group were given $0.6 \mathrm{mg}$ of glycopyrrolate intravenously, followed five minutes later by $2.5 \mathrm{mg}$ of neostigmine (subgroups $H G$ and $E G$ ). The remaining two subgroups were given a mixture of glycopyrrolate $0.6 \mathrm{mg}$ and neostigmine $2.5 \mathrm{mg}$ given as a rapid intravenous injection (subgroups HGN and EGN). Continuous ECG recordings were obtained during the reversal, at a paper speed $r_{i} 25 \mathrm{~mm} / \mathrm{sec}^{-1}$. Heart rate was determined by measuring R-R interval on ECG strips, and blood pressure was recorded, by auscultation of Korotkoff's sounds using an am sphygmomanometer once every minute for ten minutes after initial injection of glycopyrrolate or the injection of the glycopyrrolate-neostigmine mixture. Administration of the halogenated anaesthetic was continued during the reversal process and other nonsurgical stimulation, such as suction of oropharyngeal secretions, was avoided during the period when changes in heart rate and blood pressure were being recorded. Mechanical ventilation was maintained until the end of the study to maintain $\mathrm{PaCO}_{2}$ between $30-40$ torr (confirmed by arterial blood gas analysis). ECG strips were studied for changes in heart rate and rhythm during the reversal process. Each patient's heart rate prior to injection of medications is referred to as the baseline heart rate, while the maximum increase from this rate during the study period is referred to as tachycardia. The difference between the baseline heart rate and the minimum heart rate recorded after neostigmine is labelled bradycardia.

Baseline heart rates and maximum changes in heart rates (tachycardia and bradycardia) from each subgroup were compared with all other subgroups using one-way analysis of variance to determine statistical significance. Specific differences between groups were sought using Tukey's method for multiple comparisons. ${ }^{9}$

\section{Results}

Table II shows the heart rates (mean \pm S.D.) at the beginning of reversal (baseline heart rate), maximum increase (tachycardia), and decrease (bradycardia) following injections of glycopyrrolate and 
TABLE II Changes in heart rate during reversal of neuromuscular block (mean \pm S.D. beats $/$ min)

\begin{tabular}{|c|c|c|c|}
\hline \multirow[b]{2}{*}{ Sub-group } & \multirow{2}{*}{$\begin{array}{l}\text { Base-line } \\
\text { heart rate }\end{array}$} & \multicolumn{2}{|c|}{ Changes in heart rate } \\
\hline & & Tachycardia & Bradycardia \\
\hline HG & $77 \pm 15.7$ & $26 \pm 8.1$ & $0.75 \pm 10.1$ \\
\hline EG & $88 \pm 12.4$ & $10 \pm 6.3$ & $8.1 \pm 7.2$ \\
\hline $\mathrm{HGN}$ & $81 \pm 15.2$ & $9 \pm 9.4$ & $18.3 \pm 11.9$ \\
\hline EGN & $75 \pm 12.7$ & $8 \pm 10.5$ & $8.2 \pm 10.3$ \\
\hline ANOVA p-value & 0.15 & 0.001 & 0.0013 \\
\hline
\end{tabular}

Statistical analysis - no significant difference in baseline heart rates

Tachycardia

HG vs. EG - p $<0.01$

HGN vs. EGN - NS

HG vs. HGN $-\mathrm{p}<0.01$

EG vs. EGN - NS

Bradycardia

HG vs. EG - NS

HGN vs. EGN - NS

HG vs. HGN $-p<0.01$

EG vs. EGN - NS

neostigmine. Mean baseline heart rate varied between 75 to 88 . Differences between the four groups were not statistically significant $(p=0.15)$. The observed changes in heart rate during the ten-minute study period are presented in the Figure.

Tachycardia: Heart rate increased in every patient after injection of glycopyrrolate given either separately or in the mixture with neostigmine. An increase in heart rate could be noticed within one minute after injection in all subgroups. In subgroup $\mathrm{HG}$, a rapid rise was seen from one to three minutes, although heart rate continued to rise slowly until five minutes, when the injection of neostigmine was given. In subgroup $\mathrm{EG}$, the rise in heart rate was spread equally over the first five minutes. The difference in tachycardia between subgroups HG and EG is highly significant $(\mathrm{p}<0.01)$. On the other hand, in subgroups HGN and EGN, the heart rate increased for the first two minutes only and then started decreasing. The difference between subgroups HGN and EGN was not significant. Methods of administration of glycopyrrolate and neostigmine (whether given separately or simultaneously) made a significant difference only in patients who received halothane anaesthesia as the difference in tachycardia was significant only between two halothane subgroups (HG vs. HGN, p $<0.01$ ) and not between enflurane subgroups (EG vs. EGN).

Bradycardia: Administration of neostigmine was accompanied by slowing of heart rates in all patients. No significant difference was seen between respective halothane and enflurane sub- groups (HG vs. EG and HGN vs. EGN). The mode of administration of glycopyrrolate and neostigmine (whether given separately or simultaneously) did not make any difference in patients anaesthetized with enflurane (EG vs. EGN) while in those given halothane anaesthesia significantly lower heart rates were observed when glycopyrrolate and neostigmine were administered simultaneously (HG vs. HGN: $p<0.01$ ). Changes in blood pressurc paralleled those in heart rate. In subgroup HGN, additional glycopyrrolate had to be administered in two patients after heart rates below $50 / \mathrm{min}$ were observed at the end of the study period.

Cardiac rhythm: Transient junctional tachycardia was noted very rarely (one case in subgroup HG and one in EGN), lasted for less than one minute and did not produce any significant haemodynamic effects requiring treatment.

\section{Discussion}

Fluctuation of heart rate during the reversal of neuromuscular blockade has been a major concern

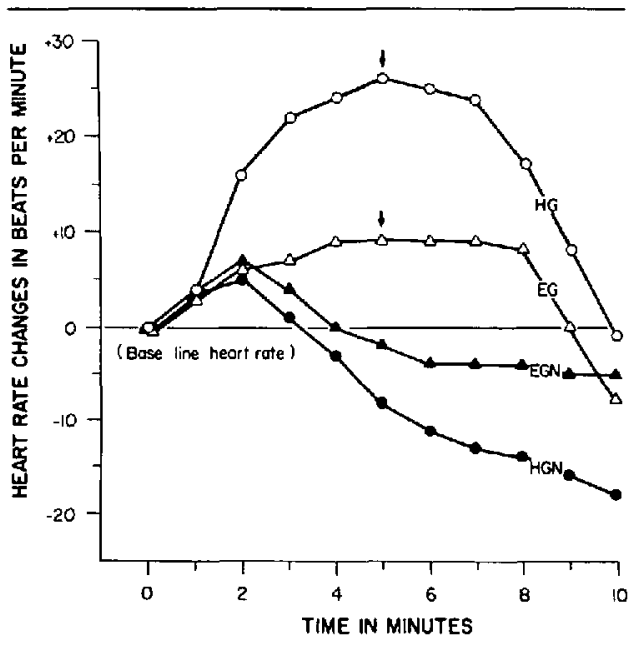

FIGURE 1 Heart rate changes in beats per minute. Baseline heart rate in each subgroup is expressed as 0 . Increase and decrease from this heart rate (beats/min) during the study period are plotted. Arrows indicate time of injection of neostigmine in sub-groups HG and EG.

HG = glycopyrrolate preceded neostigmine during halothane anaesthesia. HGN = glycopyrrolate and neostigmine given simultaneously. $\mathrm{EG}=$ glycopyrrolate preceded neostigmine during enflurane anaesthesia. EGN = simultaneous injection of atropine and glycopyrrolate during enfiurane unaesthesia. 
of the anaesthetist ever since neostigmine has been employed for this purpose. Extreme bradycardia or even cardiac arrest on the one hand, and alarming tachycardia on the other, have been observed and some cases have been reported in the literature. ${ }^{10,11}$ At one time many anaesthetists actually recommended that nondepolarizing muscle relaxants should not be reversed at all because of the problems of fluctuations of the heart rate. Not surprisingly, therefore, numerous studies have been undertaken to investigate techniques which will reduce the incidence and amount of these fluctuations. ${ }^{12-14}$ Anticholinesterases other than neostigmine (e,g., pyridostigmine and edrophonium) have been introduced ${ }^{3-5}$ which have been claimed to produce a minimum chronotropic effect on the heart and different anticholinergics (e.g., glycopyrrolate) have been introduced ${ }^{1.2}$ with a claim of providing better protection against the dangerous fluctuations of heart rate during the reversal process. In fact, it is now generally believed that glycopyrrolate causes less tachycardia than atropine and that because of its longer action, it more consistently prevents bradycardia after the anticholinesterase medications. ${ }^{13}$

A recent report ${ }^{8}$ has shown that during reversal of neuromuscular blockade utilizing atropine with neostigmine, the fluctuations in the heart rate are significantly more noticeable if the patient has been anaesthetized with halothane rather than enflurane. Since this observation is of great clinical importance, we thought it is important to find if glycopyrrolate used in similar circumstances would produce different results. Even when glycopyrrolate has been substituted for atropine (either given separately or mixed with neostigmine), we saw significantly more tachycardia in patients who received halothane anaesthesia compared to those who received enflurane. However, our data suggest that mode of administration of glycopyrrolate and neostigmine (given separately or simultaneously) has a significant effect on increase in heart rate in patients anaesthetized with halothane, but not in those given enflurane anaesthesia. Under halothane anaesthesia administration of glycopyrrolate prior to neostigmine leads to significantly greater increase in heart rate, but heart rate following neostigmine remains closer to baseline. When neostigmine and glycopyrrolate are administered simultaneously, rise in heart rate is less, but ten minutes later heart rates are slower than baseline and may necessitate administration of more glycopyrrolate.

Thus our study confirmed the findings of Samra et al. that halothane and enflurane per se have a strong modifying effect on the heart rate changes during the reversal process, irrespective of the anticholinergic agent used. Both choice of anaesthetic agent and mode of administration of anticholinergic and anticholinesterase medication have a significant effect on chronotropic changes during reversal of neuromuscular block.

The mechanism of this interaction is not clear. However, it is known that different volatile anaesthetics act quite differently on the SA node and conductive system of the heart. ${ }^{15-17}$ Bosnjak and Kampine $^{18}$ have found that atropine produces identical effects on sinus rate during halothane, enflurane or isoflurane anaesthesia. Most of these observations were obtained during animal experimentation. Obviously, further studies are required to clarify the exact mechanism of this clinically important interaction.

\section{References}

1 Ramamurthy $S$, Shaker $M N$, Winnie AP. Glycopyrrolate as a substitute for atropine in neostigmine reversal of muscle relaxant drugs. Can Anaesth Soc. J 1972; 19: 399-411.

2 Oduro KA. Glycopyrrolate methobromide, 2. Comparison with atropine sulphate in anaesthesia. Can Anaesth Soc J 1975; 22: 466-73.

3 Fogdall RP, Miller RD. Antagonism of d-tubocurarine and pancuronium induced neuromuscular blockades by pyridostigmine in man. Aneschesiology $1973 ; 39: 504-9$.

4 Ferguson A, Egerszegi P, Bevan DR. Neostigmine, pyridostigmine and edrophonium as antagonists of pancuronium. Anesthesiology 1980; 53: 390-4.

5 Agar I, Phaim AN, Karambekar D, Lear E. The heart rate following edrophonium-atropine and edrophonium-glycopyrrolate mixtures. Anesthesiology 1983; 59: 39-41.

6 Samra SK, Cohen PJ. Modification of chronotropic response to anticholinergics by halogenated anaesthetics in children. Can Anaesth Soc J 1980; 27: $540-5$.

7 Munchow $O B$, Denson JS. Modification by light cyclopropane and halothane anesthesia of the 
chronotropic effect of atropine in man. Anesth Analg 1965; 44: 782-90.

8 Samra SK, Pandit UA, Pandil SK, Kothary SP. Modification by halogenated anesthetics of chronotropic response during reversal of neuromuscular blockade. Can Anaesth Soc J 1983; 30: 48-52.

9 Neter J, Wasserman W. Applied linear statistical methods. Ist Ed. Illinois: Richard D. Irwin, Inc. (1974).

10 Mclntosh RR. Death following injection of neostigmine. Br Med J 1949; 1: 852.

11 Hill $M$. Death after injection of neostigmine. $\mathrm{Br}$ Med J 1949; 2: 601 .

12 Ovassapain A. Effects of administration of atropine and neostigmine in man. Anesth Analg 1969; 48: 219-23

13 Mirakhur RK, Dundee JW. Jones CJ, Coppel DL, Clark RSJ. Reversal of neuromuscular blockade: Dose determination studies with atropine and glycopyrrolate given before or in a mixture with neostigmine. Anesth Analg 1981; 60: 557-62.

14 Ostheimer GW. A comparison of glycopyrrolate and atropine during reversal of non-depolarizing neuromuscular block with neostigmine. Anesth Analg 1977; 56: 182-6.

15 Reynolds AK, Chiz JF, Pasquet AF. Halothane and methoxyflurane - A comparison of their effects on cardiac pace-maker fibers. Anesthesiology 1970; 33: 602-10.

16 Krisna G, Paradise $R$. Mechanism of chronotropic effects of volatile inhalation anesthetics. Anesth Analg 1977; 56: 173-81.

17 Pratila MG, Pratilas V. Anesthetic agents and cardiac electromechanical activity. Anesthesiology 1978; 49: 338-60.

18 Bosnjak ZJ, Kampine JP. Effects of halothane, enflurane and isoflurane on S.A. node. Anesthesiology $1982 ; 58: 314-21$.
Résumé

Dans cette étude portant sur 48 malades, on a observé l'influence de l'halothane et de l'enflurane sur les changements de fréquence cardiaque qui suivent la neutralisation du bloc neuromusculaire. La prémédication et la technique anesthêsique étaient les mêmes, seul l'agent principal variait: halothane ou enfurane. Le bloc neuromusculaire était neutralisé par l' injection de glycopyrrolate et néastigmine administrés séparément ou simultanément. Des changements de fréquence statistiquement significatifs ont été observés d'un groupe à l'autre: les patients du groupe enfurane présentant moins de fluctuations des fréquences cardiaques. L'injection simultanée de glycopyrrolute et de néostigmine a contribué à diminuer la tachycardie dans les sous-groupes à l'halothane seulement. 